\title{
Rôle de CREB dans la dépendance à la morphine
}

L'administration prolongée de morphine à l'animal ou à l'homme entraîne des phénomènes de dépendance complexes. On peut distinguer : (1) une dépendance physique, avec perturbation des fonctions autonomes, dans laquelle le locus cœeruleus joue un rôle important; (2) des modifications psychiques et comportementales liées aux projections de l'aire tegmento-ventrale vers le noyau accumbens; (3) un renforcement d'associations des systèmes de mémoire qui prédispose aux rechutes. On connaît relativement bien le mécanisme des actions aiguës, analgésiantes, de la morphine. Au niveau du locus cœruleus, par exemple, cette action se manifeste par une diminution des décharges nerveuses liée à l'ouverture de canaux $\mathrm{K}^{+}$et à l'inhibition des canaux $\mathrm{Na}^{+}$et $\mathrm{Ca}^{2+}$. L'action sur les canaux $\mathrm{K}^{+}$passe par les protéines Gi et Go; celle sur les canaux $\mathrm{Na}^{+}$est indirecte et résulte d'une déphosphorylation, elle-même dépendante d'une inhibition de l'activité adénylyl cyclasique (via $\mathrm{Gi}$ ). Les effets au long cours de la morphine sont beaucoup plus difficiles à expliquer. Un seul modèle existe, proposé il y a plus de 21 ans par Klee, Sharma et Nirenberg (Bethesda, MD, USA) [1] et il est toujours d'actualité. Il implique une hypersensibilité réactionnelle du système de synthèse de l'AMP cyclique, conduisant à une augmentation de la phosphorylation du canal $\mathrm{Na}^{+}$, ainsi qu'à une augmentation de la synthèse et de la phosphorylation des synapsines, le tout rendant compte de l'hyperexcitabilité neuronale (voir [2] pour une revue).

Quelles sont les preuves expérimentales à l'appui de ce modèle? Les études de Klee, Sharma et Nirenberg [1] ont été effectuées sur des cellules hybrides rat-souris NG 108-15, relativement riches en récepteurs $\delta$ des opiacés. L'effet chronique de la morphine y est relativement difficile à mettre en évidence. C'est ainsi que l'activité adénylyl cyclasique est augmentée de $100 \%$ au maximum, et habituellement de $30 \%$ [2], après un traitement de quelques heures des cellules à la morphine, lui-même suivi par une incubation plus courte avec l'antagoniste naloxone.

Plus récemment, le groupe de Z. Vogel (Jérusalem, Israël) a trouvé des résultats analogues, mais beaucoup plus nets, en raison du grand nombre de récepteurs, sur des cellules CHO qui ont été transfectées de manière stable avec le récepteur $\mu$ des opiacés [3]. Alors qu'un traitement aigu par la morphine entraîne une inhibition complète de l'accumulation d'AMP cyclique dans ces cellules, une préincubation de quatre heures avec de la morphine (ou l'analogue spécifique DAMGE), suivie par un lavage en présence de naloxone, se traduit par une augmentation de $400 \%$ de l'accumulation d'AMP cyclique.

Le mécanisme moléculaire de ces observations n'est pas clair. Le récepteur n'est pas désensibilisé, l'activité phosphodiestérasique ne semble pas impliquée et, dans l'étude de Vogel, la cycloheximide n'empêche pas le phénomène d'hypersensibilisation, ce qui tendrait à éliminer le rôle d'une augmentation de la synthèse protéique. Néanmoins, l'absence d'anticorps spécifique de chacune des isoformes de l'adénylyl cyclase (il en existe 9 à l'heure actuelle [4]) rend difficile une appréciation exacte des modifications possibles de leur synthèse. D'ailleurs, nous avons montré précédemment par hybridation in situ qu'un traitement in vivo par la morphine chez la souris entrấnait en 12 à 36 heures une augmentation de la quantité d'ARNm codant pour l'adénylyl cyclase de type 8 dans le locus cœeruleus [5].

Quoi qu'il en soit, ces résultats, retrouvés de manière constante depuis 20 ans, plaident en faveur d'un rôle de l'AMP cyclique dans le maintien de l'état de dépendance. $\mathrm{Si}$ c'est bien le cas, on devrait pouvoir interrompre cet état en agissant sur l'une des étapes-clés de l'action intracellulaire de l'AMP cyclique, le facteur transcriptionnel CREB dont la phosphorylation et l'activation dépendent de la sous-unité catalytique de la protéine kinase dépendante de l'AMP cyclique. C'est ce que nous avons exploré, en collaboration avec les groupes de $\mathrm{B}$. Roques (Inserm U. 266, Paris) et G. Schütz (DKFZ, Heidelberg), chez des souris qui ont subi une invalidation de leurs gènes codant pour les isoformes $\alpha$ et $\Delta$ de CREB [6]. Chez de telles souris, le nombre de récepteurs des opiacés n'est pas modifié; la morphine conserve son action analgésique, mais tous les symptômes physiques de l'état de dépendance (sauts, tremblements, ptose, diarrhée...) sont très nettement atténués. Il faut, bien sûr, indiquer que plusieurs isoformes de CREB existent et que l'invalidation complète de toutes ces formes en même temps est incompatible avec la survie des souris. Dans le cas présent, seules les isoformes $\alpha$ et $\Delta$ de CREB ont été invalidées alors que l'isoforme $\beta$ est plutôt augmentée [7]. Au total on peut penser que $10 \%$ à $20 \%$ 
seulement de l'activité CREB subsistent. A noter aussi que l'activité CREM est augmentée, peut-être de manière compensatoire [8]. Il s'agit là de reserves importantes, mais qui n'enlèvent pas sa valeur à la conclusion que la protéine CREB joue un rôle-clé dans la mise en place et le maintien du syndrome physique de manque après administration de morphine. Il faut rappeler à ce propos que CREB joue aussi un rôle majeur dans un autre type d'adaptation neuronale, la consolidation de la mémoire à long terme, chez les invertébrés comme chez les mammifères ( $m / s n^{\circ}$ 5, vol. 8, p. 491; $n^{\circ} 8$, vol. 11, p. 1175) $[9,10]$.

J.H.

1. Sharma SK, Klee WA, Nirenberg M. Dual regulation of adenylate cyclase accounts for narcotic dependence and tolerance. Proc Natl Acad Sci USA 1975; 72: 3092-6.

2. Noël F, Iourgenko V, Pouille Y, Hanoune J. Approches moléculaires de l'action des opiacés. médecine/sciences 1994; 10: 116-26.

3. Avidor-Reiss T, Bayevitch M, Levy R, MathusLeibovitch N, Nevo I, Vogel Z. Adenylyl cyclase supersensitization in $\mu$-opioid receptor-transfected chines hamster ovary cells following chronic opioid treatment. J Biol Chem 1995; 270 : 29732-8. 4. Hanoune J. Les adénylyl cyclases des mammifères. médecine/sciences 1994; 10: 444-7.

5. Matsuoka I, Maldonado R, Defer N, Noël F, Hanoune J, Roques BP. Chronic morphine administration causes region-specific increase of brain type VIII adenylyl cyclase mRNA. European J Pharmacol 1994; 268: 215-21.

6. Maldonado R, Blendy JA, Tzavara E, Gass P, Roques BP, Hanoune J, Schütz G. Reduction of morphine abstinence in mice with a mutation in the gene encoding CREB. Science 1996; 273: 657-9.

7. Blendy JA, Kaestner KH, Schmid W, Gass P, Schütz G. Targeting of the CREB gene leads to up-regulation of a novel CREB mRNA isoform. EMBO J 1996; 156: 1098-106.

8. Hummler E, Cole TJ, Blendy JA, Ganss R, Aguzzi A, Schmid W, Beermann F, Schütz G. Targeted mutation of the CREB gene: compensation within the CREB/ATF family of transcription factors. Proc Natl Acad Sci USA 1994; 91 : 5647-51.

9. Frank DA, Greenberg ME. CREB: a mediator of long-term memory from mollusks to mammals. Cell 1994; 79: 5-8.

10. Peschanski M. La transgenèse à la découverte des mécanismes de la mémoire. médecine/sciences $1992 ; 8: 870-1$

\section{BRÈVES}

Connexion entre la voie des MAP kinases et la régulation transcriptionnelle par l'AMP cyclique. La voie principale de transmission du signal issue des facteurs de croissance passe par les petites protéines G p21 Ras, Raf, MEK et MAPK [1]. La MAP kinase activée phosphoryle des protéines kinases activées sur la protéine ribosomique $\mathrm{S} 6$, la $\mathrm{p} 90^{r s k}$, l'activant à son tour. Ces deux kinases sont alors transférées dans le noyau. La MAP kinase phosphoryle le facteur TCF/Elk-1, un partenaire de SRF (serum response factor) qui intervient dans la stimulation transcriptionnelle de gènes appelés «à réponse ultraprécoce » dont l'archétype est le gène c-fos. Ce gène, essentiel aux événements ultérieurs associés à la prolifération cellulaire et à la réponse inflammatoire, est également stimulé par l'AMP cyclique car son promoteur contient un site de fixation pour le facteur CREBP (cyclic AMP response element-binding factor). Cependant, le mécanisme activant CREBP en réponse à un facteur de croissance n'était pas connu. Xing et al. (Boston, MA, Baltimore, MD, USA) répondent à cette question dans un récent article de Science. Ils montrent en effet que p90 ${ }^{\text {sk }}$ est en réalité une CREB-kinase, capable de phosphoryler CREB sur la sérine 133, qui est également la cible de la PKA (protéine kinase activée par l'AMP cyclique) [2]. Cependant, l'article publié simultanément par Nakajima et al. du laboratoire de Marc Montminy (Harvard Medical
School, Boston, MA, USA) dans la revue Cell amène à se demander si cette phosphorylation de CREB par p90 $0^{r k}$ peut réellement activer des gènes sensibles à l'AMP cyclique [3]. En effet, ces auteurs notent que p90 ${ }^{r s k}$ activé par l'insuline ou le NGF (nerve growth factor), via la voie Ras et les MAP-kinases, forme un complexe avec CBP (CREB binding protein, $m / s n^{\circ} 10$, vol 12, p. 1113) en interagissant avec elle au niveau de son site de liaison au produit de l'oncogène adénoviral $E 1 A$. Or, les actions de E1A et de p90 rsk complexés à CBP sont identiques: au lieu de l'activer, ils inhibent la réponse à l'AMP cyclique, probablement en empêchant l'interaction de CREB avec CBP, indispensable à l'activation transcriptionnelle. Les auteurs démontrent également que la formation du complexe $\mathrm{CBP} / \mathrm{p} 90^{r s k}$ est indispensable aux effets différenciateurs du NGF sur des cellules PC12, et suggèrent que ce mécanisme pourrait expliquer en partie l'antagonisme entre l'insuline et les hormones stimulant la synthèse d'AMP cyclique sur la transcription des gènes du métabolisme, tel celui de la phosphoénolpyruvate carboxykinase, codant pour une enzyme de la gluconéogenèse.

[1. Kahn A. médecine/sciences 1992; $8: 1097-9$.

[2. Xing J, et al. Science 1996; 273: 959-63.]

[3. Nakajima T, et al. Cell 1996; 86: 465-74.] 